\title{
A mathematical model approach to a glycerolysis reaction for monoacylglycerol production
}

\author{
B. Cheirsilp \& A. H-Kittikul \\ Department of Industrial Biotechnology, Faculty of Agro-Industry, \\ Prince of Songkla University, Hat Yai, Thailand
}

\begin{abstract}
Monoacylglycerol emulsifiers commonly employed in the food, cosmetic and pharmaceutical industries can be produced by a glycerolysis reaction using lipase. The concentrations of two substrates (palm olein and glycerol) in the glycerolysis reaction were strictly interdependent. Therefore, it was impossible to perform a classic kinetic study by fixing the concentration of one substrate and changing the concentration of the other substrate. Here, a mathematical model approach is a useful tool to independently assess the effects of hypothetical changes in the concentrations of each. In essence, this analysis permits one to examine various aspects associated with the dynamics and equilibrium of the glycerolysis reaction that cannot be investigated experimentally. In this study a mathematical model, taking into account the mechanism of the glycerolysis reaction for monoacylglycerol production using immobilized lipase, has been developed. From the proposed model, the effects of varying the initial concentrations of substrates on the initial production rate and yield of monoacylglycerol were simulated. The most significant finding from simulation was that an increase in the initial concentration of triacylglycerol leads to an increase in the initial production rate of monoacylglycerol, but there is a limit beyond which increasing the initial concentration of triacylglycerol results in a low yield of monoacylglycerol. The simulation results show that mostly glycerol reacts with fatty acid of triacylglycerol to produce monoacylglycerol in a glycerolysis reaction. From a thermodynamic standpoint, a greater incorporation of glycerol is expected because a higher concentration of this acyl acceptor should shift the equilibrium towards a greater glycerolysis reaction.

Keywords: glycerolysis, lipase, mathematical model, monoacylglycerol.
\end{abstract}




\section{Introduction}

The applications of glycerolysis reaction using lipase have been carried out to produce monoacylglycerol due to its mild reaction conditions and position specific products [1-3]. The concentrations of two substrates, triacylglycerol and glycerol, in glycerolysis reaction were strictly interdependent. To identify the optimal conditions for lipase catalyzed glycerolysis reaction, it is essential to understand the kinetics of this reaction. Until now all kinetic mechanisms on lipase catalytic reactions are only based on hydrolysis of triacylglycerol [4,5] or esterification of fatty acid [6,7]. Only a limited number of kinetic studies for glycerolysis using glycerol as acyl acceptor have been found in literature [1]. However, the reported model is rather complicated and narrow range of application. Here, in this paper the kinetics of glycerolysis of palm olein for monoacylglycerol production was studied. First, a simple model based on PingPong $\mathrm{Bi} \mathrm{Bi}$ was proposed to describe the kinetics of hydrolysis and esterification steps involved in glycerolysis reaction. Then, the mathematical model for glycerolysis reaction was considered. The effects of enzyme, water, glycerol and palm olein concentrations on monoacylglycerol production were contributed in the model. The constructed model was used to obtain a better understanding of the effects of two substrates, triacylglycerol and glycerol in glycerolysis reaction. Finally, optimal condition was determined by simulation study using the model.

\section{Materials and methods}

\subsection{Materials}

Lipase PS (Pseudomonas sp.) was a gift from Amano Pharmaceutical Co. Ltd., Japan. Microporous polypropylene powder; Accurel EP-100 (particle size 200$400 \mu \mathrm{m}$ ) was a gift from Akzo Nobel (Obermburg, Germany). Palm olein was purchased from Morakot Industry Co. Ltd., Thailand. All other chemicals were analytical grade reagents obtained from commercial sources.

\subsection{Immobilization}

To immobilize lipase, Accurel EP-100 (10g) was added to $100 \mathrm{ml}$ of $0.1 \mathrm{M}$ phosphate buffer ( $\mathrm{pH} 7$ ) containing lipase PS approximately $100 \mathrm{U} / \mathrm{ml}$ and the reaction mixture was stirred with a magnetic bar at $100 \mathrm{rpm}$ for $30 \mathrm{~min}$. Afterward, $100 \mathrm{ml}$ of $0.1 \mathrm{M}$ phosphate buffer $(\mathrm{pH} 7)$ was added and the suspension was filtered through a filter paper by vacuum. The immobilized lipase PS on Accurel EP-100 (330 U/g) was stored at $4{ }^{\circ} \mathrm{C}$ for further studies.

\subsection{Glycerolysis reaction}

The glycerolysis experiments were carried out in batch system. The reaction mixture consisted of various enzyme, water, glycerol and palm olein concentrations in organic solvent (acetone/isooctane mixture 3:1, v/v). The 
temperature was controlled at $45{ }^{\circ} \mathrm{C}$. The reaction mixture was mixed by magnetic stirrer at $300 \mathrm{rpm}$. Samples of the reaction mixture were centrifuged to remove immobilized lipase before analysis.

\subsection{Analytical method}

The components of oil phase were analyzed for triacylglycerol (TAG), diacylglycerol (DAG), monoacylglycerol (MAG) and fatty acid (FA) using a thin-layer chromatography with flame ionization detection (TLC/FID) (IATROSCAN MK5, Iatron Laboratories Inc. Tokyo, Japan). In this experiment, percent of peak area was assumed as percent content of the corresponding compound. Activity of lipase was determined by the modified cupric acetate method. One unit of hydrolytic activity was defined as the amount of the enzyme, which liberates $1 \mu \mathrm{mol}$ equivalent of palmitic acid from palm olein in 1 $\min$ at $30^{\circ} \mathrm{C}$.

Ordinary differential equations were solved by the Runge-Kutta single-step fourth-order method [8]. The programs were coded in the Visual Basic program ver. 6.0 (Microsoft Inc., USA).

(a)

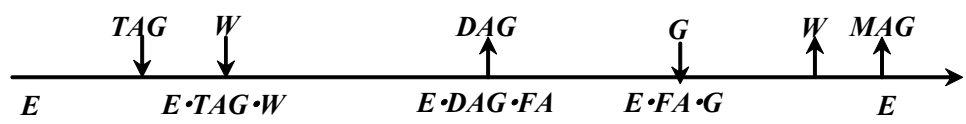

(b)

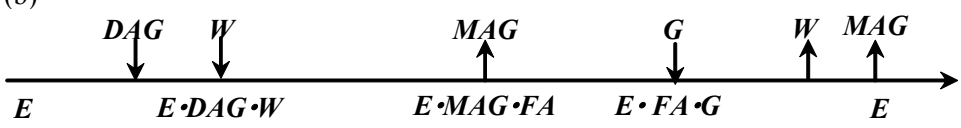

Figure 1: Schematic diagram of Ping Pong $\mathrm{Bi} \mathrm{Bi}$ mechanisms for glycerolysis reaction of triacylglycerol (a) and diacylglycerol (b). $T A G, D A G, M A G, W, G$ and $E$ denote triacylglycerol, diacylglycerol, monoacylglycerol, water, glycerol and enzyme, respectively. $E \cdot T A G \cdot W, E \cdot D A G \cdot W, E \cdot D A G \cdot F A, E \cdot M A G \cdot F A$ and $E \cdot F A \cdot G$ are different complexes between enzyme and the species defined above.

\section{Results and discussion}

\subsection{Modeling of glycerolysis reaction}

Mechanisms of glycerolysis reaction for monoacylglycerol production were interesterifications of tri- or di-acylglycerol with excess glycerol. The interesterification reaction involves sequential execution of the hydrolysis and reesterification steps, and thus requires multiple entrances and exits of reactant and product species in such a manner as to render the overall mechanism of the 
Ping-Pong type as in Fig. 1. Theoretically glycerolysis of one mole of triacylglycerol and two moles of glycerol could produce 3 moles of monoacylglycerol, however the yields of monoacylglycerol depend on flavored equilibrium in various conditions [9]. In kinetic study of immobilized enzyme, the reaction rate might be controlled by an internal mass transfer. However, it was also reported that mass transfer limitation in porous supports could be neglected [10,11]. Therefore, to describe a simple mathematical model for glycerolysis reaction, mass transfer limitation in reaction system was first neglected. The appearance rates of triacylglycerol $(T A G)$, diacylglycerol $(D A G)$, monoacylglycerol $(M A G)$, glycerol $(G)$, fatty acid $(F A)$ and water $(W)$ from hydrolysis and reesterification steps were derived as in Table 1.

Table 1: A mathematical model of glycerolysis reaction.

\begin{tabular}{|c|c|}
\hline Differential equations & \\
\hline$d[T A G]-\left(-V_{m D A G}[T A G][W]+V_{r D A G}[D A G][F A]\right) E_{T}$ & (1) \\
\hline$d t=\overline{\left([W]+K_{m D A G}[D A G]+K_{m M A G}[M A G]+K_{m G}[G]\right)[F A]}$ & \\
\hline$\underline{d[D A G]}=\underline{\left(V_{m D A G}[T A G][W]+V_{r M A G}[M A G][F A]-V_{m M A G}[D A G][W]-V_{r D A G}[D A G][F A]\right) E_{T}}$ & (2) \\
\hline$\frac{d t}{d t}=\frac{\left([W]+K_{m D A G}[D A G]+K_{m M A G}[M A G]+K_{m G}[G]\right)[F A]}{2}$ & \\
\hline 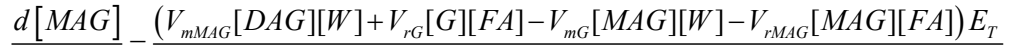 & (3) \\
\hline$d t \quad\left([W]+K_{m D A G}[D A G]+K_{m M A G}[M A G]+K_{m G}[G]\right)[F A]$ & \\
\hline$\left(V_{m G}[M A G][W]-V_{r G}[G][F A]\right) E_{T}$ & (4) \\
\hline$\overline{d t}-\overline{\left([W]+K_{m D A G}[D A G]+K_{m M A G}[M A G]+K_{m G}[G]\right)[F A]}$ & \\
\hline$\underline{d[F A]}=\underline{\left(V_{m D A G}[T A G][W]+V_{m M A G}[D A G][W]+V_{m G}[M A G][W]\right) E_{T}}$ & $(5)$ \\
\hline$\left([W]+K_{m D A G}[D A G]+K_{m M A G}[M A G]+K_{m G}[G]\right)[F A]$ & \\
\hline$-\frac{\left(V_{r D A G}[D A G][F A]+V_{r M A G}[M A G][F A]+V_{r G}[G][F A]\right) E_{T}}{\left([W]+K_{m D A G}[D A G]+K_{m M A G}[M A G]+K_{m G}[G]\right)[F A]}$ & \\
\hline$\underline{d[W]}=\underline{\left(V_{r D A G}[D A G][F A]+V_{r M A G}[M A G][F A]+V_{r G}[G][F A]\right) E_{T}}$ & (6) \\
\hline$d t \quad\left([W]+K_{m D A G}[D A G]+K_{m M A G}[M A G]+K_{m G}[G]\right)[F A]$ & \\
\hline$-\frac{\left(V_{m D A G}[T A G][W]+V_{m M A G}[D A G][W]+V_{m G}[M A G][W]\right) E_{T}}{\left([W]+K_{m D A G}[D A G]+K_{m M A G}[M A G]+K_{m G}[G]\right)[F A]}$ & \\
\hline
\end{tabular}

$F A$ : fatty acid concentration, $W$ : water concentration, $E_{T}$ : total enzyme concentration. $V_{m D A G}$ and $V_{r D A G}$ are maximum initial reaction rates for hydrolysis of $T A G$ and reesterification of $D A G$ defined as: $V_{m D A G}=\frac{k_{1} k_{3}}{k_{2}+k_{3}}$ and $V_{r D A G}=\frac{k_{4} k_{6}}{k_{4}+k_{5}}$, respectively. $K_{m D A G}, K_{m M A G}$ and $K_{m G}$ are equilibrium constants for $D A G, M A G$ and $G$ defined as: $K_{m D A G}=\frac{k_{5}}{k_{6}}, K_{m M A G}=\frac{k_{11}}{k_{12}}$ and $K_{m G}=\frac{k_{17}}{k_{18}}$, respectively. $V_{m M A G}$ and $V_{r M A G}$ are maximum initial reaction rates for hydrolysis of $D A G$ (production of $M A G$ ) and reesterification of $M A G$ defined as: $V_{m M A G}=\frac{k_{7} k_{9}}{k_{8}+k_{9}}$ and $V_{r M A G}=\frac{k_{10} k_{12}}{k_{10}+k_{11}}$, respectively. $V_{m G}$ and $V_{r G}$ are maximum initial reaction rates for hydrolysis of $M A G$ (production of $G$ ) and reesterification of $G$ defined as: $V_{m G}=\frac{k_{13} k_{15}}{k_{14}+k_{15}}$ and $V_{r G}=\frac{k_{16} k_{18}}{k_{16}+k_{17}}$, respectively. 
The concentrations of triacylglycerol, diacylglycerol, monoacylglycerol and fatty acid at different times were obtained experimentally when the substrate concentration of triacylglycerol and glycerol were 7.16 and $19.14 \mathrm{mM}$, respectively. The concentrations of enzyme and water were $0.6 \mathrm{~g}$ and $9.89 \mathrm{mM}$, respectively. The nonlinear curve fitting by Simplex's method [12] was used for fitting the system of differential equations (1-6) (Table 1) into the experimental data. By fitting the above differential equations to the experimental data, the rate constants were estimated and listed in Table 2.

Base upon the obtained rate constants and the kinetic scheme, the concentrations of each composition at different reactions could be calculated. Comparison between calculated and experimental data was presented in Fig. 2 and a good agreement was obtained.

In terms of the reaction rate constants in Table 2, the forward reaction rate constants in the first and second reactions $\left(V_{m D A G}=0.115 \mathrm{mM}^{-1} \mathrm{~g}^{-1} \mathrm{~h}^{-1}, V_{m M A G}=\right.$ $\left.0.151 \mathrm{mM}^{-1} \mathrm{~g}^{-1} \mathrm{~h}^{-1}\right)$ were much lower than that in the third forward reactions $\left(V_{m G}\right.$ $\left.=0.811 \mathrm{mM}^{-1} \mathrm{~g}^{-1} \mathrm{~h}^{-1}\right)$. The results indicated that the first and second hydrolysis reactions ( $T A G$ to $D A G$ and $D A G$ to $M A G$ ) were the limiting steps during the overall reactions. The production rate of $M A G$ by glycerolysis reaction $\left(V_{r G}=\right.$ $\left.1.23 \mathrm{mM}^{-1} \mathrm{~g}^{-1} \mathrm{~h}^{-1}\right)$ was higher than the production rate by hydrolysis reaction of $D A G\left(V_{m M A G}=0.151 \mathrm{mM}^{-1} \mathrm{~g}^{-1} \mathrm{~h}^{-1}\right)$. These rate constants show that the intermediates $(G$ and $F A$ ) were easily converted to $M A G$ by glycerolysis reaction. The experimental results also showed that the concentration of the measurable intermediate $(F A)$ was low during time course of glycerolysis (Fig. 2).

Table 2: Rate constants in the model of glycerolysis reaction.

\begin{tabular}{lclc}
\hline $\begin{array}{l}\text { Maximum initial } \\
\text { reaction rates } \\
\left(\mathrm{mM}^{-1} \mathrm{~g}^{-1} \mathrm{~h}^{-1}\right)\end{array}$ & Value & $\begin{array}{l}\text { Equilibrium constants } \\
\left(\mathrm{mM}^{-2}\right)\end{array}$ & Value \\
\hline$V_{m D A G}$ & 0.115 & $K_{m D A G}$ & $1.09 \times 10^{-4}$ \\
$V_{m M A G}$ & 0.151 & $K_{m M A G}$ & $3.20 \times 10^{-4}$ \\
$V_{m G}$ & 0.811 & $K_{m G}$ & $1.23 \times 10^{-3}$ \\
$V_{r D A G}$ & 0.109 & & \\
$V_{r M A G}$ & 0.320 & & \\
$V_{r G}$ & 1.23 & & \\
\hline
\end{tabular}

\subsection{Effect of triacylglycerol and glycerol}

The effects of varying the initial concentrations of triacylglycerol and glycerol on the initial production rate and yield of monoacylglycerol were also simulated. Simulation of glycerolysis system under a variety of initial conditions provides a more complete picture of the dynamics and equilibrium behavior of this system (Fig. 3). Two of the most significant findings were (1) an increase in the initial 


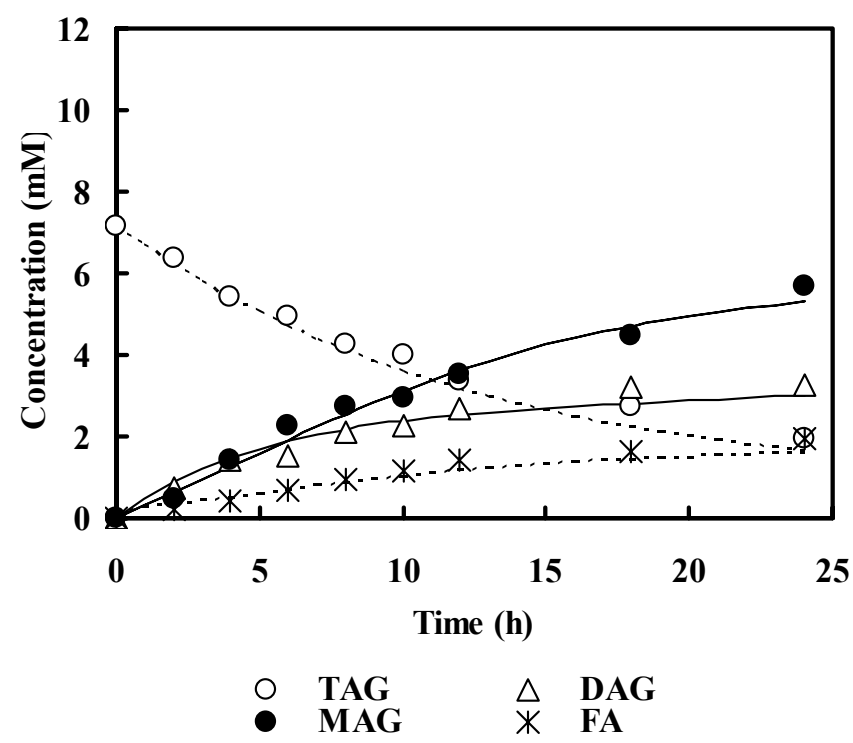

Figure 2: Comparison between calculated (lines) and experimental results (symbols) of glycerolysis reaction. Triacylglycerol $7.16 \mathrm{mM}$; glycerol $19.14 \mathrm{mM}$; water $9.89 \mathrm{mM}$; immobilized lipase $0.6 \mathrm{~g}$.
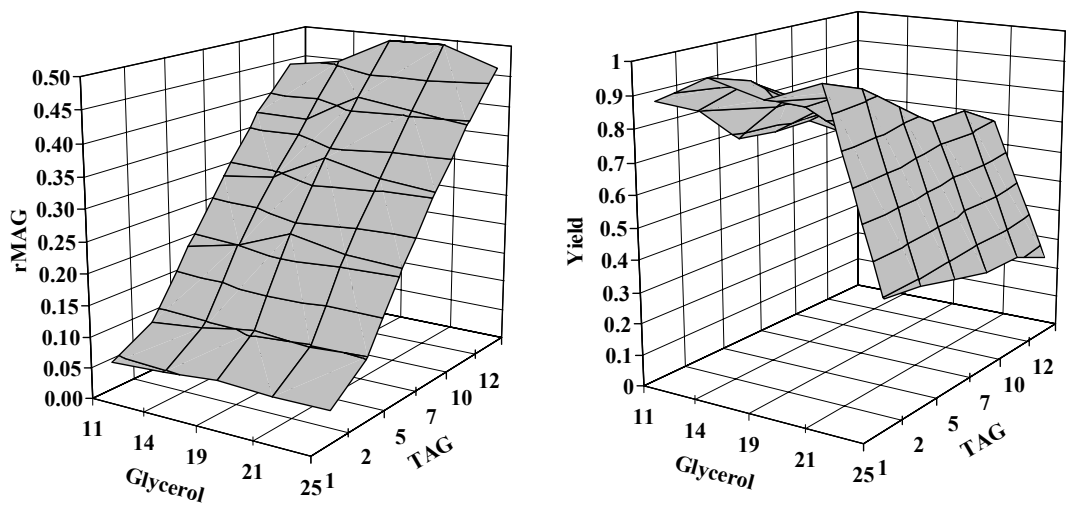

Figure 3: Simulation results of effects of triacylglycerol (TAG) and glycerol concentrations on the initial production rate (rMAG) and yield of monoacylglycerol.

concentration of triacylglycerol leads to an increase in the initial production rate of monoacylglycerol, but there is a limit beyond which increasing the initial concentration of triacylglycerol results low yield of monoacylglycerol at each glycerol concentration and (2) increasing in glycerol concentration more than 
$19.14 \mathrm{mM}$ the effect becomes less pronounced in initial production rate and yield of monoacylglycerol at equilibrium. The former result is expected from a thermodynamic standpoint, because a higher concentration of triacylglycerol should enhance the greater production rate of monoacylglycerol. The latter result might be due to the limitation of equilibrium comes from limited enzyme concentration. Therefore, it was concluded that glycerol concentration of 19.14 $\mathrm{mM}$ was the optimal concentration for glycerolysis reaction with enzyme concentration of $0.6 \mathrm{~g}$.

From all of the above, in the case of high reaction rate and acceptable yield of monoacylglycerol are set as target in process optimization, the glycerolysis reaction with high concentrations of triacylglycerol and glycerol are suitable to be carried out. On the other hand, in the case of high yield of monoacylglycerol is set as target in process optimization, low concentration of triacylglycerol and excess concentration of glycerol are suitable.

\section{Conclusion}

The kinetics of lipase-catalyzed glycerolysis reaction between triacylglycerol and glycerol were successfully modeled using rate expressions requiring adjustable parameters. The present model was effective for prediction the synergic effect of two substrates (triacylglycerol and glycerol) in glycerolysis reaction. The simulation results showed that mostly glycerol reacted with fatty acid of triacylglycerol to produce monoacylglycerol in glycerolysis reaction. From a thermodynamic standpoint, a greater incorporation of glycerol is expected because a higher concentration of this acyl acceptor should shift the equilibrium toward greater glycerolysis reaction. Another important observation was that the behavior of high triacylglycerol concentrations showed high initial production rates but low yields of monoacylglycerol.

\section{References}

[1] Tan, T. \& Yin, C., The mechanism and kinetic model for glycerolysis by 1,3 position specific lipase from Rhizopus arrhizus. Biochemical Engineering Journal, 25, pp. 39-45, 2005.

[2] McNeill, G.P., Shimizu, S. \& Yamane, T., High-yield enzymatic glycerolysis of fats and oils. Journal American Oil Chemical Society, 68(1), pp. 1-5, 1991.

[3] McNeill, G.P. \& Yamane, T., Further improvements in the yield of monoglycerides during enzymatic glycerolysis of fats and oils. Journal American Oil Chemical Society, 68(1), pp. 6-10, 1991.

[4] Taylor, F., Kurantz, M.J. \& Craig, J.C., Kinetics of continuous hydrolysis of tallow in a multi-layered flat-plate immobilized-lipase reactor. Journal American Oil Chemical Society, 69(6), pp. 591-594, 1992.

[5] Padmini, P., Rakshit, S.K. \& Baradarajan, A., Kinetics of enzymatic hydrolysis of rice bran oil in organic system. Enzyme and Microbial Technology, 16, pp. 432-435, 1994. 
[6] Zhang, T., Yang, L., Zhu, Z. \& Wu, J., The kinetic study on lipasecatalyzed transesterification of $\alpha$-cyano-3-phenoxybenzyl alcohol in organic media. Journal of Molecular Catalysis B: Enzymatic, 18, pp. 315323, 2002.

[7] Xu, Y., Du, X. \& Liu, D., Study on the kinetics of enzymatic interesterification of triglycerides for biodiesel production with methyl acetate as the acyl acceptor. Journal of Molecular Catalysis B: Enzymatic, 32, pp. 241-245, 2005.

[8] Danby, J.M.A., Computer modeling, Willmann-Bell Inc Richmond Va 1997.

[9] Reyes H.R., Kinetic modeling of interesterification reactions catalyzed by immobilized lipase. Biotechnology and Bioengineering, 43, pp. 171-182, 1994.

[10] Chen, J.P. \& Wang, H.Y., Improved properties of bilirubin oxidase by entrapment in alginate-silicate sol-gel matrix. Biotechnology Techniques, 12(11), pp. 851-853, 1998.

[11] Romero, M.D., Calvo, L., Alba, C. \& Daneshfar, A., A kinetic study of isoamyl acetate synthesis by immobilized lipase-catalyzed acetylation in n-hexane. Journal of Biotechnology, 127, pp. 269-277, 2007.

[12] Nelder, J.A. \& Mead, R., A simplex method for function minimization. Computational Journal, 7, pp. 308-313, 1964. 